\title{
Twist drill aspiration of chronic subdural hematoma at the Ignace Deen hospital of Conakry
}

\begin{abstract}
Bedside percutaneous twist drill aspiration (TDA) is described as a surgical method of management of Chronic subdural hematoma (CSDH) and appear to be a reasonable approach in low medical resources environment. We report the results of TDA of CSDH in a single tertiary centre in Conakry Republic of Guinea in West Africa, for the period March 2015 to October 2017. The charts and medical record of 38 cases of CSDH treated with TDA were collected retrospectively, with a mean follow-up of 84.2 days. The outcome data assessed were neurologic status evaluated by the follow-up Markwalder grading scale (MGS: $0=$ normal to $4=$ coma), recurrence, infection and mortality. The Male-Female ratio was 1.3:1. Mean age at diagnosis was $78.2+/-12$ years. Traumatic brain injury (TBI) was identified as causal in $28.2 \%$ of cases studied. All patients were operated on under local anesthesia and had a postoperative drain left in place for 3 days. The mean postoperative MGS was 1.06, up from a mean preoperative MGS of 3.7. Two cases of recurrence occurred subsequent to TDA causing death and three other patients died from unknown causes during the follow-up period. No cases of post-operative infection were diagnosed during the follow-up. Our study shows that Bedside TDA appears to be a suitable alternative to burr hole craniotomies in urgent cases of $\mathrm{CSDH}$ in the low socio-economic setting, where a surgical theatre is not always available.
\end{abstract}

Keywords: chronic subdural hematoma, Twist-drill aspiration, outcome
Volume 9 Issue 5 - 2019

\author{
Alpha Boubacar Bah,' Seylan Diawara,' \\ Ibrahima Sory Souare, 'Abdoulaye Barry,' \\ Ansoumane Donzo,' Joseph Denamou, ${ }^{2}$ \\ Abdoulaye Bobo Diallo' \\ 'Department of Surgery, Ignace Deen Hospital, Guinea \\ ${ }^{2}$ Department of Anesthesia, Donka Hospital, Guinea
}

Correspondence: Alpha Boubacar Bah, Neurochirurgien, Service de Chirurgie Générale, CHU Ignace Deen Kaloum, Conakry, Guinée, Tél +224 660121212,

Email cushing.bah@gmail.com

Received: August 03, 2019 | Published: September 20, 2019

\section{Introduction}

Burr-hole craniostomy (BHC) with a post-operative closed drainage system appear to be the gold standard choice of surgical treatment for chronic subdural hematoma (CSDH). ${ }^{1}$ The Twist-drill drainage procedure has been reported to be relatively safe, time-saving, and cost-effective in the treatment of CSDH and can be performed at the patient's bedside. ${ }^{2}$ It has been gaining in popularity recently due to a progressive refinement in the technique to limit classically-reported complications related to its "blind" use. ${ }^{3}$ According to a number of recent publications, TDA performed at the bedside is just as effective as BHC and likewise can safely and effectively be the first line of treatment in selected patients with symptomatic chronic subdural hematoma. ${ }^{4}$

In Guinea, there is a lack of organized neurosurgical care with adequate availability of surgical theatres, resources, and manpower for medical emergencies. There is not a single Intensive Care Unit in the entire country, and patients' families must themselves finance their relatives' medical care. Under these difficult conditions, we have been using bedside TDA as a last resort treatment for critically ill patients in need of an urgent surgical aspiration when no surgical theatre is available or financially feasible.

The aim of this case series is to evaluate the outcome of the bedside TDA for CSDH in these very ill patients treated in a hospital with poor medical resources and to compare it with the literature regarding more traditional treatment, including our previous report of CSDH predominantly treated by $\mathrm{BHC}$ in a semi-privately run hospital in the same city. ${ }^{5}$ The results may influence whether bedside TDA may be used both safely and effectively not only as a first-line treatment for
CSDH patients under extreme conditions, but also for select cases in the general population of symptomatic CSDH.

\section{Patients and method}

We retrospectively analysed data for all patients who underwent percutaneous TDA for CSDH between March 2015 and October 2017 at the department of General Surgery of the Ignace Deen Hospital in Conakry, Guinea. The study was approved by the Institutional Review Board (IRB) for that facility. Patients without a minimal follow-up of 4 weeks were excluded.

Ninety-four patients were operated on for CSDH in the institution by a single surgeon, which represent $22 \%$ of the neurosurgical case $\log$ during the study period. Of these 94 patients, 41 (43.6\%) were operated on at the bedside using the TDA technique. Three of those patients were lost to follow-up after discharge. The recorded data included age, sex, duration of symptoms in weeks, history of trauma, comorbidities, the pre- and post-operative neurologic examinations, the CT scan description (including density, septations, thickness and midline shift), and the treatment performed. With regard to the preoperative treatment method(s) used prior to performing the bedside TDA procedure, we also considered patients' recent intake of aspirin or anticoagulant medication, the perioperative medical treatment, and the number and skull site(s) of used for decompressive drilling.

The TDA procedure choice were only determined by the clinical emergency with the non-availability of a surgical theatre and was performed in the emergency bed. In non-septate CSDH, the skull was drilled on one site either the frontal curvature or the parietal curvature, whichever is closer to the maximal thickness of the hematoma. In septate $\mathrm{CSDH}$, the 2 sites were drilled. The hand drilling was 
preceded by a local anethesia and a $1 \mathrm{~cm}$ incision. The drilling was made with a 60 degree angulation to the surface of the bone aiming at the maximal thickness of the hematoma. The tip of the drill was very pointed for an easy penetration of the dura without slipping on it at this angle. We used multiple types of catheter depending on the availability of the resources and what the patient can afford. Ideally a Number 5 ventriculostomy catheter were used, or a 10 French gauge infant feeding tube. In some extreme cases with none of the preceding catheter available a Foley catheter $8 \mathrm{Ch}$ with multiple holes on the sides was used. Regardless the catheter, the guide wire used was distally non insulated (to allow coagulation of the dura before pushing the hand-drill to penetrate it) and curved (to guide the catheter in the subdural space with a decreased risk of brain parenchyma penetration). The catheter was introduced from 2 to $4 \mathrm{~cm}$ in the space depending the thickness of the hematoma on the CT scan and fixed to the scalp by a suture. The content is first allowed to flow freely by a gentle movement up and down of the catheter. When the spontaneous flow decreased, we performed gentle and abundant normal saline flushing before connecting the outside catheter end to an aspirative grenade-shaped bulb or sometime a urinary drainage bag, in which case a continuous gravity dependant drainage was kept. The Drain(s) were removed on the $3^{\text {rd }}$ post-operative day. No systematic postoperative CT scan were done in the immediate post-operative period, except for the occurrence of an adverse event. Patients were kept on Hydrocortisone, and Antibiotics for 3 days post op and antiepileptic drugs for 2 months.

The pre and post-operative neurologic status were assessed by the Markwalder Grading Scale (MGS). ${ }^{6}$ The grade 0 were defined by a neurologically intact patient; oriented patients with mild symptoms, absent or mild focal deficit were graded 1; drowsy patient with hemiparesis were graded 2; stuporous patients reacting appropriately to noxious stimuli and hemiplegia were graded 3; patient with no reaction of decerebrate - decorticate posturing were graded 4 (Table 1). Neurological outcome were classified as Good for MGS of 0 or 1 and bad for MGS of 2 to 4 at the last follow up. Other outcome parameters were recurrence, infection and mortality.

Table I The Markwalder Grading Scale ${ }^{6}$

\begin{tabular}{ll}
\hline Grade & \multicolumn{1}{c}{ Clinical description } \\
\hline 0 & Patient neurologically normal \\
I & $\begin{array}{l}\text { Patient alert and oriented; mild symptoms such as headache; absent or mild neurological deficit such as reflex } \\
\text { asymmetry }\end{array}$ \\
2 & Patient drowsy or disoriented with variable neurological deficit, such as hemiparesis \\
3 & Patient stuporous but responding appropriately to noxious stimuli; severe focal signs such as hemiplegia \\
4 &
\end{tabular}

\section{Results}

Thirty eight patients were included in the study. The mean followup were 84.2 days and the age range was $48-92$ years with a mean of $78.2 \pm 12$ years and a pic incidence in the $7^{\text {th }}$ decade group. The sex ratio Male Female was 1.3-1. A remote history of brain trauma was found in $28.9 \%$. The mean duration of the symptoms were 22 weeks ranging from 7 weeks to 48 weeks. Eleven patients $(28.9 \%)$ were on Aspirin for a presumed diagnosis of ischemic stroke. No patients were on anticoagulation medication. The mean pre-operative MGS were 3.7 and 30 patients had a MGS of 3 at the time of surgery. The comorbidities included hypertension $(92.1 \%)$, diabetes mellitus $(63.15 \%)$, and malaria $(36.84 \%)$. Six cases $(16.6 \%)$ were bilateral. The maximum thickness of the hematoma on the axial CT ranged from 16 to $36 \mathrm{~mm}$ (mean 28.1 \pm 5.7 ). A midline shift of more than $10 \mathrm{~mm}$ was present in all cases. The densities were mixed in 12 patients $(31.5 \%)$ and multiple septations were found in 7 cases $(18.4 \%)$. All patients with multiple septations received 2 twist drill hole with placement of two drain (frontal and parietal). A single twist drill was performed on the remaining patients. Post operatively the mean MGS improved to $1.06(n=33)$ at last follow-up and $68.48 \%$ of patient had good clinical outcome. Five patients died (13.5\%), 2 of them from recurrence of the hematoma after an initial improvement with a discharge home and before we get the opportunity to do a revision surgery. The other 3 patients died less than 2 days following the surgical procedure from aspiration pneumonia and sepsis and had all a pre-operative MGS of 4. No cases of infection and post-operative seizure were diagnosed. The data are summarized in the Table 2.

Table 2 Epidemiological, Clinical and Outcome data of our series

\begin{tabular}{|c|c|c|c|}
\hline & & $\mathbf{n}$ & $\%$ \\
\hline Number of patients & & 38 & \\
\hline Sex Ratio M.F & & 1.3 & \\
\hline Age $<60$ & & 0 & 0 \\
\hline 60-Age-69 & & 8 & 21.05 \\
\hline Age $>80$ & & 30 & 78.94 \\
\hline Aspirin treatment & & 11 & 28.9 \\
\hline Recent TBI & & 11 & 28.9 \\
\hline Clinical course & & & 22 weeks \\
\hline \multirow[t]{2}{*}{ Pre-op MGS } & $<3$ & 5 & 13.15 \\
\hline & $\geq 3$ & 33 & 86.84 \\
\hline Mixed Density & & 12 & 31.5 \\
\hline Mean midline shift & & & $13.42 \mathrm{~mm}$ \\
\hline Mortality & & 5 & 13.15 \\
\hline
\end{tabular}




\begin{tabular}{llll} 
Table continued & & & \\
\hline Recurrence & & $\mathbf{n}$ & $\%$ \\
Seizure & & 2 & 5.2 \\
Comorbidité & HTA & 35 & 0 \\
& Diabète & 24 & 62.1 \\
& Paludisme & 14 & 36.84 \\
MGS recul & 0 & 3 & 7.8 \\
n=33 & 1 & 23 & 60.52 \\
& 2 & 5 & 13.15 \\
& $03-A p r$ & 2 & 5.2
\end{tabular}

\section{Discussion}

The originality of this study resides in the fact that patients were selected for this specific surgical treatment solely based on the medical environment constraints: (Limited availability of a surgical theatre, No surgical Intensive Care Unit in the whole country, and lack of surgical equipment supply.....) In most reported studies and description of TDA or craniostomy, the criteria of selection were different. ${ }^{7,8}$ Mixed density, hyperdense lesions, calcified, ossified, organized and multi loculated CSDH were excluded, in which cases, a BHU or even a craniotomy with membranectomy under general anesthesia were generally preferred. The old classical twist drill drainage could be associated with inadequate drainage, brain penetration, and acute epidural hematoma. ${ }^{9}$ Recent modification of the technique and new systems have been recently reported to prevent these complications with some success, allowing TDA to gain more popularity as first line treatment of selected cases of CSDH.

The first major modification of the technique was published in 2003 by Asfora et al. ${ }^{10}$ The author used a subdural evacuating port system (SEPS) that promote brain expansion, avoid brain penetration by the use of a Port and there is no need for irrigation. The hematoma evacuation is enhanced by gradual decompression using a uniform negative extradural pressure. The technique is more effective in the hypodense subdural collections compared to mixed density collections.

In 2007 Sucu et al. ${ }^{3}$ described a series of step to further reduce the rate of brain penetration, epidural bleeding and inadequate evacuation of the hematoma: the use of a pointed tip drill bit to avoid dural separation and bleeding; Increasing the angle of skull penetration and positioning the drill posterior to the area of maximal thickness to avoid brain penetration; increasing the drill bit size to allow entry of larger diameter irrigation catheter to reduce inadequate drainage.

In 2012 Krieg et al. ${ }^{11}$ described another minimally invasive technique of placement of hollow screws under local anesthesia, but $20 \%$ of patients needed revision BHC for inadequate drainage. In the same year Yadav et al. ${ }^{12}$ described a way to coagulate the dura using an insulated guidewire, and a curved introduction of the catheter to avoid brain penetration and catheter kinking. They also advocated for a more variable choice for the site of drilling either at the frontal or the parietal curve. But inadequate drainage was a limitation, which occurred in $14 \%$ of cases. The definition of inadequate drainage by most of these authors is mainly based on post-operative CT scan finding, not clinical signs. Poor correlation of radiological and clinical outcome of CSDH are well documented in the literature. ${ }^{13,14}$ In our series the need for a post-operative CT scan likewise was driven by the occurrence of an adverse clinical event.

In 2014 Chari et al. ${ }^{15}$ did a systematic review to collate and analyse the published experience with these modified TDA techniques in comparison with other surgical methods. ${ }^{15}$ Nine eligible studies were found comprising 796 patients treated with these novel techniques. Pooled analysis showed a success rate of $77 \%$, recurrence rate of $22.4 \%$ and in hospital mortality of $1.4 \%$.

Qing-Feng, Wang et al. ${ }^{16}$ introduced a new modified TDC technique using a novel device, the YL-1 puncture needle, and evaluated its efficacy and advantages compared with BHC. A retrospective study involving 121 patients with $\mathrm{CSDH}$ who underwent surgery was conducted, involving 68 patients undergoing modified TDC and 53 patients treated by BHC. The neurological and radiological outcomes did not differ significantly between the TDC and BHC groups, while the rates of complication and pneumocrania in patients who underwent the modified TDC were significantly lower than that in those who underwent BHC. The recurrence and reoperation rates in patients from the 2 groups were similar. The operation duration and length of hospital stay of the patients who underwent the modified TDC were significantly shorter than those of the patients who underwent BHC.

\section{Conclusion}

The surgical techniques we used in our patients are derived from a synthesis of all these techniques in addition to more irrigation of the subdural space and a longer duration of post-operative drainage than reported in the literature to reduce the risk of inadequate evacuation. Despite the differences in the criteria of selection of patients between these studies and ours, the epidemiological profile of our patients are comparable: Older patients in the $7^{\text {th }}$ decade, with multiple comorbidities, and acute decompensation. This is in contrast with another population of chronic subdural hematoma that can be found in Guinea, as previously reported by the same team, a younger population of patient with a higher rate of neglected TBI history, a shorter clinical course and obviously a more satisfactory outcome with BHC. ${ }^{5}$

Nevertheless, we obtained an improvement of the mean MGS from 3,7 to 1.06 . The mortality rate is higher than reported in other studies but is closely related to the poor pre-operative neurological status, the lack of post-operative ICU for MGS grade 4 patients and the delay we had in operating recurrent cases coming from home.

Our study is limited by the small number of patients and cannot be compared to our previous report of $\mathrm{CSDH}$ treated with $\mathrm{BHC}$ due to the different population, different hospital setting and different criteria of selection. Even with more sophisticated study design in developed countries, Class I evidence on the best surgical treatment for CSDH is still missing. However, the current study results do support the conclusion that bedside TDA is a valid alternative for the safe and effective treatment of CSDH and can be used as first line treatment in elderly patients ideally with hypodense hematoma on the CT. In the low socio-economic environment, no patient should be denied the oportunity to be surgically treated, based on criteria like advanced age, multiple comorbidities and non-availability of a surgical theatre. A bedside TDA with the described modified safety precautions can clinically improve most of these patients with reasonably low complication rates. 


\section{Acknowledgments}

None.

\section{Conflicts of interest}

The author declares no conflicts of interest.

\section{Funding}

None.

\section{References}

1. Lega BC, Danish SF, Malhotra NR, et al. Choosing the best operation for chronic subdural hematoma: A decision analysis. J Neurosurg. 2010;113(3):615-621.

2. Santos-Ditto RA, Santos-Franco JA, Pinos-Gavilanes MW, et al. Management of chronic subdural hematoma with twist-drill craniostomy. Report of 213 patients. Gac Med Mex. 2007;143(3):203-208.

3. Sucu HK, Gokmen M, Ergin A, et al. Is there a way to avoid surgical complications of twist drill craniostomy for evacuation of a chronic subdural hematoma? Acta Neurochir (Wien). 2007;149(6):597-599.

4. Horn EM, Feiz-Erfan I, Bristol RE, et al. Bedside twist drill craniostomy for chronic subdural hematoma: a comparative study. Surg Neurol. 2006;65(2):150-153.

5. Bah AB, Souare IS, Diawara S, et al. Traitement chirurgical des hématomes sous duraux chroniques en Guinée: Étude de 22 cas à l'hôpital Kipe de Conakry. Neurochirurgie. 2019;65(2-3):83-88.

6. Markwalder TM. Chronic subdural hematomas: A review. J Neurosurg. 1981;54(5):637-645.

7. Kenning TJ, Dalfino JC, German JW, et al. Analysis of the subdural evacuating port system for the treatment of subacute and chronic subdural hematomas. J Neurosurg. 2010;113(5):1004-1110.
8. Reinges $\mathrm{MH}$, Hasselberg I, Rohde V, et al. Prospective analysis of bedside percutaneous subdural tapping for the treatment of chronic subdural haematoma in adults. J Neurol Neurosurg Psychiatry. 2000;69(1):40-47.

9. Reinges MH, Rübben A, Spetzger U, et al. Minimally invasive bedside craniotomy using a selfcontrolling pre-adjustable mechanical twist drill trephine. Surg Neurol. 1998;50(3):226-229.

10. Asfora WT, Schwebach L. A modified technique to treat chronic and subacute subdural hematoma: Technical note. Surg Neurol. 2003;59(4):329-232.

11. Krieg SM, Aldinger F, Stoffel M, et al. Minimally invasive decompression of chronic subdural haematomas using hollow screws: efficacy and safety in a consecutive series of 320 cases. Acta Neurochir. 2012;154(4):699-705.

12. Yadav Y, Yadav S, Parihar VS. Modified Twist Drill Technique in the Management of Chronic Subdural Hematoma. Turk Neurosurg. 2013;23(1):50-54.

13. Lin X. Comparing twist-drill drainage with burr hole drainage for chronic subdural hematoma. Chinese Journal of Traumatology. 2011;14(3):170-173.

14. Asghar M, Adhiyaman V, Greenway MW, et al. Chronic subdural haematoma in the elderly - a North Wales experience. $J R$ Soc Med. 2002;95(6):290-292.

15. Chari A, Kolias AG, Santarius T, et al. Twist-drill craniostomy with hollow screws for evacuation of chronic subdural hematoma. $J$ Neurosurg. 2014;121(1):176-183.

16. Qing-Feng Wang, Cheng Cheng, Chao You. A New Modified Twist Drill Craniostomy Using a Novel Device to Evacuate Chronic Subdural Hematoma. Medicine (Baltimore). 2016;95(10):e3036. 